\title{
ON THE MAPPING OF QUADRATIC FORMS ${ }^{1}$
}

\author{
LLOYD L. DINES
}

The development of this paper was suggested by a theorem proposed by Bliss, proved by Albert, ${ }^{2}$ by Reid, ${ }^{3}$ and generalized by Hestenes and McShane. ${ }^{4}$ That theorem had to do with two quadratic forms $P(z)$ and $Q(z)$ in real variables $z^{1}, z^{2}, \cdots, z^{n}$ with real coefficients, and may be stated as follows:

If $P(z)$ is positive at each point $z \neq(0)$ at which $Q(z)=0$, then there is a real number $\mu$ such that the quadratic form $P(z)+\mu Q(z)$ is positive definite. ${ }^{5}$

If one considers the set of points $\mathfrak{M}$ in the $x y$-plane into which the $z$-space is mapped by the transformation

$$
x=P(z), \quad y=Q(z),
$$

he will note that the above theorem may be interpreted as asserting the existence of a supporting line of the map $\mathfrak{M}$ which has contact with $\mathfrak{M}$ only at $(x, y)=(0,0)$. This suggests that the theorem is related to the theory of convex sets.

In the present paper it is proven (Theorem 1) that $\mathfrak{M}$ is a convex set. Furthermore it is proven (Theorem 2) that if $P(z)$ and $Q(z)$ have no common zero except $z=(0)$, then $\mathfrak{M}$ is closed, and is either the entire $x y$-plane or an angular sector of angle less than $\pi$. Immediate corollaries include not only the theorem quoted above, but also statements of criteria for the existence of (1) semi-definite, and (2) definite linear combinations $\lambda P(z)+\mu Q(z)$. The author hopes in a subsequent paper to obtain analogous results for the general case of $m$ quadratic forms.

Throughout the paper it is to be understood without further statement that $P(z)$ and $Q(z)$ are quadratic forms in $z^{1}, z^{2}, \cdots, z^{n}$, with real coefficients, the variables $z^{i}$ being restricted to real values.

1. Convexity, and the condition for $\lambda P(z)+\mu Q(z) \geqq 0$. We give first the following theorem.

1 Presented to the Society, December 31, 1940.

2 This Bulletin, vol. 44 (1938), p. 250.

3 This Bulletin, vol. 44 (1938), p. 437.

4 Transactions of this Society, vol. 47 (1940), p. 501.

5 While the present paper was in press, Professor N. H. McCoy kindly called the author's attention to the fact that this theorem was proven first by Paul Finsler: Über das Vorkommen definiter und semidefiniter Formen in Scharen quadratischer Formen, Commentarii Mathematici Helvetici, vol. 9 (1937), pp. 188-192. Apparently this work had been overlooked by the authors referred to above. 
THEOREM 1. Under the transformation (1), the map $\mathfrak{M}$ of the z-space onto the $x y$-plane is convex.

If $A$ is a point of the map, distinct from the origin $O$, every point of the ray $O A$ belongs to the map, since $P(r z)=r^{2} P(z)$ and $Q(r z)$ $=r^{2} Q(z)$ for every real number $r$. Hence, if $A$ and $B$ are two points collinear with $O$, and each belongs to $\mathfrak{M}$, then all points of the line segment $A B$ belong to $\mathfrak{M}$.

We will therefore assume that $A\left(x_{1}, y_{1}\right)$ and $B\left(x_{2}, y_{2}\right)$ are points of $\mathfrak{M}$, not collinear with the origin, defined by

$$
\begin{array}{ll}
x_{1}=P\left(z_{1}\right), & x_{2}=P\left(z_{2}\right), \\
y_{1}=Q\left(z_{1}\right), & y_{2}=Q\left(z_{2}\right), \quad z_{i}=\left(z_{i}^{1}, z_{i}^{2}, \cdots, z_{i}^{n}\right),
\end{array}
$$

and attempt to show that every point on the line segment $A B$ belongs to $\mathfrak{M}$. Without loss of generality we will further assume that

$$
x_{2} y_{1}-x_{1} y_{2}=k^{2}>0 .
$$

It will suffice to show analytically that if $\bar{t}$ is any given number such that $0<\bar{t}<1$, then the equations

$$
P(z)=x_{1}+\bar{t}\left(x_{2}-x_{1}\right), \quad Q(z)=y_{1}+\bar{t}\left(y_{2}-y_{1}\right)
$$

admit a real simultaneous solution $z=\left(z^{1}, z^{2}, \cdots, z^{n}\right)$.

In (4) we make the substitution

$$
z=\rho\left(z_{1} \cos \theta+z_{2} \sin \theta\right)
$$

where $\rho$ and $\theta$ are real variables, and write the results in the form

$$
\begin{aligned}
& \rho^{2} p(\cos \theta, \sin \theta)=x_{1}+\bar{t}\left(x_{2}-x_{1}\right), \\
& \rho^{2} q(\cos \theta, \sin \theta)=y_{1}+\bar{t}\left(y_{2}-y_{1}\right),
\end{aligned}
$$

where $p$ and $q$ are quadratic forms in $\cos \theta, \sin \theta$, defined by

$$
\begin{aligned}
p(\cos \theta, \sin \theta) & \equiv P\left(z_{1} \cos \theta+z_{2} \sin \theta\right), \\
q(\cos \theta, \sin \theta) & \equiv Q\left(z_{1} \cos \theta+z_{2} \sin \theta\right) .
\end{aligned}
$$

Elimination of $\rho^{2}$ from the two equations (6) imposes upon $\theta$ the condition

$$
y_{1} p(\cos \theta, \sin \theta)-x_{1} q(\cos \theta, \sin \theta)=\bar{t} T(\theta)
$$

where

$$
T(\theta) \equiv\left(y_{1}-y_{2}\right) p(\cos \theta, \sin \theta)-\left(x_{1}-x_{2}\right) q(\cos \theta, \sin \theta) .
$$

The function $T(\theta)$ is a quadratic form in $\cos \theta, \sin \theta$, which has the positive value $k^{2}$ at $\theta=-\pi / 2, \theta=0$, and $\theta=\pi / 2$; as can be easily veri- 
fied from (7), (2), and (9). Since it can vanish for at most two values of $\theta$ between $-\pi / 2$ and $\pi / 2$, and must be negative between any two such values if they exist, the function $T(\theta)$ will be positive on at least one of the two intervals $-\pi / 2 \leqq \theta \leqq 0$ or $0 \leqq \theta \leqq \pi / 2$. We will suppose, for definiteness, that it is the latter, the argument being similar in the two cases.

We define a function $f(\theta)$ by the formula

$$
f(\theta)=\frac{y_{1} p(\cos \theta, \sin \theta)-x_{1} q(\cos \theta, \sin \theta)}{T(\theta)}, \quad 0 \leqq \theta \leqq \pi / 2,
$$

which is obviously continuous on the range indicated, and which has the further properties $f(0)=0$ and $f(\pi / 2)=1$. Hence it takes on all values between 0 and 1 , and in particular there is a value $\bar{\theta}$ such that $f(\bar{\theta})=\bar{t}$. This $\bar{\theta}$ is then a solution of $(8)$.

The compatibility condition (8) being satisfied by $\theta=\bar{\theta}$, we easily satisfy the two equations (6) by taking $\rho^{2}=\bar{\rho}^{2}=k^{2} / T(\bar{\theta})$. And the resulting

$$
z=\bar{z}=\bar{\rho}\left(z_{1} \cos \bar{\theta}+z_{2} \sin \bar{\theta}\right)
$$

given by (5) provides the required solution for (4).

Corollary. A necessary and sufficient condition that there exist real $\lambda, \mu$, such that for all real $z$

$$
\lambda P(z)+\mu Q(z) \geqq 0
$$

is that there exist real $a, b$, such that the two equations $P(z)=a, Q(z)=b$ are inconsistent for real $z$.

The condition is necessary, since in its absence the map $\mathfrak{M}$ is the entire $x y$-plane, and every line $\lambda x+\mu y=0$ separates the plane into a positive half-plane and a negative half-plane, each of which contains points determined by $x=P(z), y=Q(z)$.

However, if the point $(a, b)$ does not belong to the map, no point on the ray from the origin to $(a, b)$ belongs to the map. Hence the origin is a boundary point of the convex set $\mathfrak{M}$, and through this boundary point there passes a supporting line $\lambda x+\mu y=0$, such that $\lambda P(z)+\mu Q(z) \geqq 0$ for all real $z$.

2. Closure, and the conditions for $\lambda P(z)+\mu Q(z)>0$. We now prove the following theorem.

Theorem 2. If $P(z)$ and $Q(z)$ have no common zero except $z=(0)$, then $\mathfrak{M}$ is closed as well as convex, and is either the entire $x y$-plane or an angular sector of angle less than $\pi$. 
Since $\mathfrak{M}$ is convex, if it is not the entire $x y$-plane it lies entirely in some half-plane

$$
a x+b y \geqq 0, \quad a^{2}+b^{2}=1 .
$$

We first show that, under the stated hypothesis, $\mathfrak{M}$ cannot contain both rays of the boundary line $a x+b y=0$. Suppose it did contain the two symmetrical points $A(b,-a), B(-b, a)$, and more explicitly that

$$
P\left(z_{1}\right)=b, \quad Q\left(z_{1}\right)=-a, \quad P\left(z_{2}\right)=-b, \quad Q\left(z_{2}\right)=a .
$$

Since either $a$ or $b$ is certainly different from zero, we may assume the notation so chosen that $a>0$. Then $Q\left(z_{1}\right)<0$ and $Q\left(z_{2}\right)>0$. Hence ${ }^{6}$ there are, in the hyperplane defined by $z=z_{1} u+z_{2} v$, two linearly independent points $z_{0}=z_{1} u_{0}+z_{2} v_{0}, z_{0}^{\prime}=z_{1} u_{0}^{\prime}+z_{2} v_{0}^{\prime}$, such that

$$
Q\left(z_{0}\right)=Q\left(z_{0}^{\prime}\right)=0 .
$$

Consider now the quadratic form

$$
\phi(u, v)=a P\left(z_{1} u+z_{2} v\right)+b Q\left(z_{1} u+z_{2} v\right)
$$

in the two real variables $u, v$. It is easily verified that $\phi$ vanishes at $(u, v)=(1,0)$ and at $(u, v)=(0,1)$. These, together with the dependent points $(c, 0)$ and $(0, c)$, are its only possible zeros unless it vanishes identically. It does not vanish identically, since it does not vanish at $\left(u_{0}, v_{0}\right)$ or $\left(u_{0}^{\prime}, v_{0}^{\prime}\right)$ in view of (11) and our hypothesis. Hence, by (10), $\phi(u, v)>0$ except at $(c, 0)$ and $(0, c)$. This is clearly impossible, and the contradiction proves that the map $\mathfrak{M}$ cannot contain both points $A(b,-a)$ and $B(-b, a)$.

We now let $X(x, y)$ denote any point of $\mathfrak{M}$, and consider the angle $A O X$, where $A \equiv A(b,-a)$ and $O \equiv O(0,0)$. Then $\cos A O X$ $=(b x-a y) /\left(x^{2}+y^{2}\right)^{1 / 2}$. And as the point $z$ varies over the unit hypersphere $\|z\|=1, \cos A O X$ is represented by the function

$$
\psi(z)=\frac{b P(z)-a Q(z)}{\left[P^{2}(z)+Q^{2}(z)\right]^{1 / 2}}, \quad\|z\|=1 .
$$

In view of the hypothesis, $\psi(z)$ is continuous on this hypersphere; and since its values are bounded below by -1 and above by +1 , it attains a minimum value $m \geqq-1$ and a maximum value $M \leqq 1$. It is impossible that $m=-1$ and $M=1$, since then the map $\mathfrak{M}$ would contain both points $A(b,-a)$ and $B(-b, a)$. Hence $\mathfrak{M}$ consists of a closed

\footnotetext{
${ }^{6}$ Reference may be made to Bôcher, Introduction to Higher Algebra, p. 151, Theorem 2.
} 
sector bounded by rays $O A^{\prime}$ and $O B^{\prime}$ such that $\cos A O A^{\prime}=M$ and $\cos A O B^{\prime}=m$. And angle $A^{\prime} O B^{\prime}<$ angle $A O B=\pi$.

Corollary 1. Necessary and sufficient conditions that there exist real $\lambda, \mu$, such that for all real $z \neq(0)$

$$
\lambda P(z)+\mu Q(z)>0
$$

are that: (1) there exist real $a, b$, such that the two equations $P(z)=a$, $Q(z)=b$ are inconsistent for real $z$; and $(2) P(z)$ and $Q(z)$ have no common zero except $z=(0)$.

The necessity is obvious. The sufficiency follows from Theorem 2 . For if $(\lambda, \mu) \neq(0,0)$ is a point of $\mathfrak{M}$ on the bisector of its angular sector, then (12) is satisfied.

Corollary 2. (Bliss-Albert theorem.) If, whenever $Q(z)=0$ and $z \neq(0), P(z)>0$; then there exists a real number $\mu$ such that $P(z)+\mu Q(z)$ is positive definite.

The conditions of Corollary 1 are obviously satisfied with $(a, b)$ $=(-1,0)$. Hence there exist $\lambda, \mu$, satisfying (12). If $Q(z)$ actually vanishes for some $z \neq(0), \lambda$ is necessarily positive and hence may be taken equal to 1 .

If, on the contrary, $Q(z)$ is definite, then the map $M$ is a closed sector of which only the vertex $(0,0)$ is on the $x$-axis. Hence there is a line $x+\mu y=0$ such that $x+\mu y>0$ for all points of $\mathfrak{M}$ except $(0,0)$. Then $P(z)+\mu Q(z)$ is positive definite.

It is perhaps worthy of note that the two conditions of Corollary 1 are completely independent. This is shown by the following four examples.

Example 1, in which both (1) and (2) are satisfied:

$$
P(u, v) \equiv u^{2}, \quad Q(u, v) \equiv v^{2} .
$$

Example 2, in which (1) is satisfied but (2) is not:

$$
P(u, v) \equiv u^{2}, \quad Q(u, v) \equiv u v .
$$

Example 3, in which (1) is not satisfied but (2) is:

$$
P(u, v) \equiv u^{2}+2 u v, \quad Q(u, v) \equiv 2 u v+v^{2} .
$$

Example 4, in which neither (1) nor (2) is satisfied:

$$
P(u, v, w, t) \equiv u^{2}+2 u v+w^{2}, \quad Q(u, v, w, t) \equiv 2 u v+v^{2}+w t .
$$

Carnegie Institute of Technology 